\title{
Improved Technique of PAPR Reduction Combined Pre-distortion in OFDM Systems
}

\author{
Zhinian Luo \\ College of Information Science and Engineering \\ Hunan University \\ Changsha, China \\ zhinianluo@hnu.com
}

\author{
Jian Shi \\ College of Information Science and Engineering \\ Hunan University \\ Changsha, China \\ liaomingshijian@126.com
}

\section{CLIPPING AND FILTERING}

\begin{abstract}
Orthogonal Frequency Division Multiplexing (OFDM) is an effective parallel multi-carrier transmission scheme. However, this technique has one weakness of a higher transmission signal Peak to Average Power Ratio (PAPR) when the signal is transmitted. To reduce the impact of High Power Amplifier (HPA) nonlinearity on OFDM system, this paper proposes the combination of improved clipping and filtering method and based on digital pre-distortion lookup table reduce the peak to average ratio of OFDM and improve the BER performance of the power amplifier in pre-distortion system and the effectiveness of HPA. The simulation results show there is a certain relationship between the PAPR reduction techniques and HPA linearization techniques.
\end{abstract}

Keywords-predistortion; PAPR reduction; OFDM; power amplifier;

\section{INTRODUCTION}

Orthogonal Frequency Division Multiplexing (OFDM) is a high spectral efficiency multi-carrier modulation technique, with good resistance to multipath fading capabilities and advantages of a strong anti-multipath interference, and can achieve high-speed data transmission [1]. It has been widely applied to many areas of wireless communications in recent years. For the future fourthgeneration mobile communication systems research, OFDM technology is also to be a very promising candidate. However, the output multi-carrier OFDM system is the superposition of a plurality of sub-channel signal. If more than one signal phase is consistent, the superimposed signal's instantaneous power will be far higher than the average power of the signal. Also there is a higher peak to average power ratio (PAPR) [2] and high technical requirements' system linearization. Therefore, the high power amplifier (HPA) must have a larger linear range, otherwise it will lead to serious nonlinear distortion. Reducing PAPR of OFDM signal and solving linear power amplifier are the current major problem. Studies have shown that when the HPA works closely or over saturated region, the using of digital adaptive pre-distortion causes performance degradation [3], then it can use PAPR reduction techniques to solve the problem.
Clipping and filtering that is an effective method for reducing PAPR in OFDM system [4] directly uses the threshold value for limiting the time domain signal. If the time-domain signal is formula (1), then the original signal and a time domain signal with the clipping processing has the following relationship (2).

$$
S_{n}=\left|S_{n}\right| e^{j \theta}
$$

$$
S_{n}=\left\{\begin{array}{ll}
A e^{j \theta} & \left|S_{n}\right|>A \\
S_{n} & \left|S_{n}\right| \leq A
\end{array} \quad n=0,1,2 \ldots\right.
$$

Where $\mathrm{A}$ is the maximum amplitude of the signal system and $\theta$ is the phase of the signal. The clipping ratio $\mathrm{CR}$ is defined as follows (3).

$$
C R=\frac{A}{\sqrt{p_{i n}}}
$$

Where $p_{i n}$ represents the average power before clipping OFDM signal.

\section{B. Clipping and filtering}

Some paper mentioned the improved method of clipping and filtering is composed by the two parts about the time domain and frequency domain filtering [5], effectively improved the spread spectrum band and in-band distortion caused by the direct method of clipping. The process of the algorithm of clipping and filtering is shown in Fig1.

The implementation process firstly uses OFDM frequency domain of input data $\mathrm{L}$ times oversampling (Inserting the intermediate data $\mathrm{N}$ into (L-1) * $\mathrm{N}$ zeroes) and then transforms OFDM frequency domain signal into the

\section{A. Clipping}


time domain after the IFFT conversion of a $\mathrm{L} * \mathrm{~N}$ points, then converted to the time domain signal after clipping operation, lastly carried with FFT and external zero and IFFT to realize frequency domain filtering operation after clipping of the signal. After treatment of the above process, the system can effectively reduce the PAPR value and eliminate clipping band interference caused.

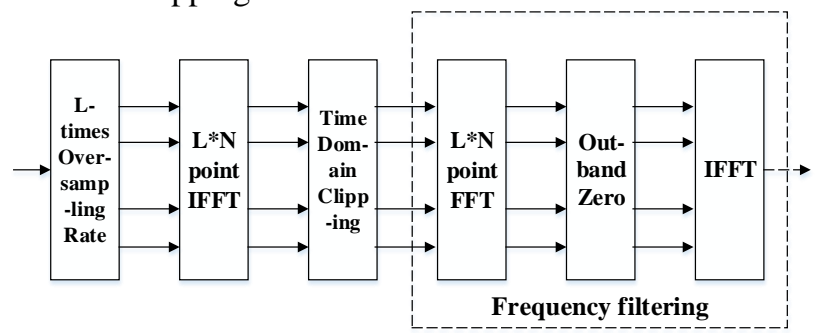

Figure 1. Clipping and filtering

\section{PREDISTORTION}

Pre-distortion is a distortion placed in front of a nonlinear amplifier unit to compensate for non-linear power amplifier. Both characteristic curves is complementary, to form linear amplification.

\section{A. Saleh model}

In order to study non-linear high power amplifiers for signal distortion, we need to establish the similar of actual operating characteristics. Saleh's memory-less Traveling Wave Tube Amplifier (TWTA) model [6] is taken into consideration in this paper, widely used in most literatures for HPA performance analysis. If we represent the complex envelope of the input signal to HPA as (4)

$$
x(t)=\rho(t) \exp [j \varphi(t)]
$$

And the output signal of HPA can be expressed by as follows (5).

$$
z(t)=A[\rho(t)] \exp [j(\varphi(t)+\phi[\rho(t)])]
$$

Where $\rho(t)$ and $\phi(\rho)$ represent AM-AM and AM-PM distortion of the nonlinear amplifier respectively. TWTA is widely used in satellite communications [7]. Its distortion characteristics can be modeled as follows (6) and (7).

$$
\begin{aligned}
& A[\rho(t)]=A_{\text {sat }} \times \frac{\rho(t)}{\rho(t)+A_{\text {sat }}^{2}} \\
& \Phi[\rho(t)]=\frac{\pi}{3} \times \frac{\rho^{2}(t)}{\rho^{2}(t)+A_{\text {sat }}^{2}} \mathrm{rad}
\end{aligned}
$$

\section{B. Digital baseband pre-distortion lookup table}

Digital baseband pre-distortion lookup table (LUT) is currently the most popular pre-distortion linearization techniques. The technology can be applied to any waveform diagram of the power amplifier gain and have a good linearization effect, the basic steps take the amplifier's input power (or amplitude, etc.) as a look-up table's index pointer, and then serve the HPA's complex-coupled as a pointer content stored in the look-up table, then find the corresponding pre-adjusted value in accordance with the amplitude value of the input information signal, and outputs it to the subsequent circuit, achieving the purpose of linearization. Simple block diagram about the algorithm of LUT pre- distortion can be figured as follows Fig 2. In Fig.2, where $\mathrm{H}[$.] represents the transform function of predistortion, the LMS algorithm [8]which estimates the LUT can be expressed as formula (8) and (9).

$$
H_{R}\left[\rho_{n}\right]_{n+1}=H_{R}\left[\rho_{n}\right]_{n}-\alpha\left(\frac{A\left[r_{n}\right]}{\rho_{n}}-1\right)
$$

$$
H_{\psi}\left[\rho_{n}\right]_{n+1}=H_{\psi}\left[\rho_{n}\right]_{n}-\beta\left(\psi_{n}-\varphi_{n}\right)
$$

Where $H_{R}$ and $H_{\psi}$ represent the information about predistorter amplitude and phase. Adjustment of coefficient $\alpha$, $\beta$ determines the rate of convergence and stability of the algorithm. This iterative algorithm converges very slowly, and because of OFDM symbol distribution characteristics, the probability of a significant signal appears relatively small, resulting in a small number of iterations, will greatly affect the convergence rate.

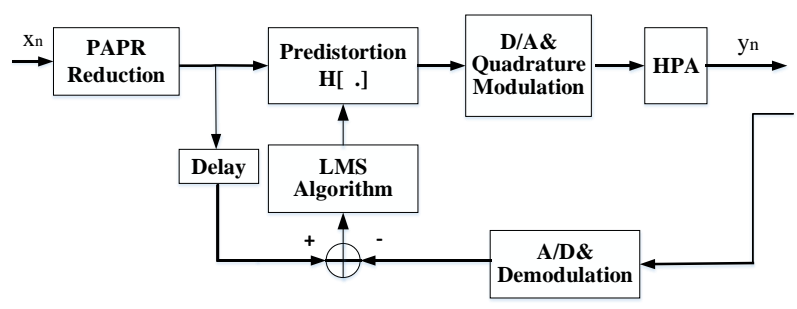

Figure 2. PAPR reduction and pre-distortion

\section{CLIPPING AND PREDISTORTION}

If the nonlinear distortion was suppressed by only clipping and filtering in OFDM systems, it causes serious in-band distortion and out-of-band interference as well as clipping. Pre-distortion technology could compensate the HPA inherent nonlinearity effectively. It could eliminate amplitude distortion and phase distortion caused by the power amplifier. The output voltage value that corresponds to the input voltage in the linear range is greater than the maximum output value of power amplifier, the nonlinear 
distortion will be not compensated by the pre-distortion technology.

\section{A. Clipping and predistortion}

Through the study of pre-distortion technology and clipping and filtering method, it found that the two methods which could reduce the nonlinear distortion are complementary:(1) clipping and filtering method, with the decrease threshold, the system reduces the value, the nonlinear distortion will be reduced, but the error rate of the system will increase, the system implementation is simple. (2) digital baseband pre-distortion method, pre-distorter allows the maximum input value, which determines the scope of the pre-distortion. When the power of the input signal is greater than the threshold, it is impossible to achieve pre-distortion's function.

\section{B. Specific implementation process}

So this paper proposes that the adaptive pre-distortion techniques and suppressing PAPR technology are combined, that is, a union approach that reduce the nonlinear distortion in OFDM systems. Specific implementation process as follows (1) After the source signal are modulated by quadrature amplitude modulation (QAM) and OFDM modulation [9], Shown in Fig.3, when the amplifier is operating in the nonlinear region, it could not be corrected directly by using pre-distortion technology, the middle section uses pre-distortion technology, when the input signal is small, the signal input was enlarged by the amplifier directly. (2) After clipping, the input signal reaches predistortion, according to the lookup table, first compensating for the amplitude of the signal by pre-distortion value, and then making the appropriate phase rotation, it is the output of the pre-distorter .3Signal were amplified after passing the high power amplifier (HPA), due to the influence of amplifier's nonlinear distortion [10], the output signal of HPA is a distortion signal. (4)After the output signal were attenuated, it is inputted to the lookup table, and it were compared with the input signal that a delay, based on the updated formula to calculate the value of the pre-distortion amplitude table and phase distortion table. (5)In cycles until the pre-distorter converged, it will compensate the nonlinear distortion of amplifier well.

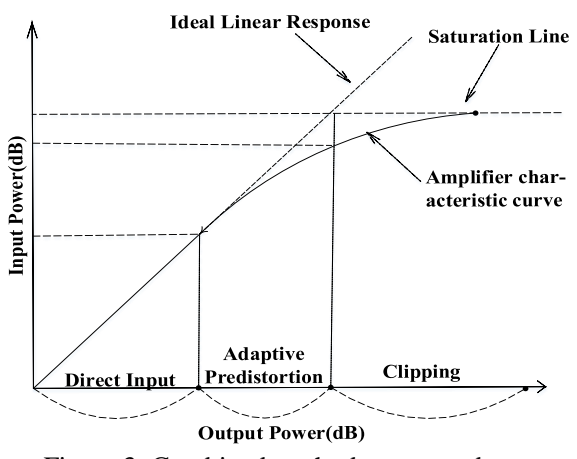

Figure 3. Combined method segmented range

\section{SIMULATION RESULTS}

In the simulation, the parameters are set as below

a) Number of parallel channel to transmit: 128

b) Channel: AWGN

c) The capacity of LUT: 512

d) Modulation level: 16QAM

e) FFT length: 128

f) HPA: Memory-less Saleh model

\section{A. Constellation}

Combined clipping and pre-distortion receiving end of the constellation gets as Fig 4. Firstly, the improved clipping makes the amplitude of the input signal not exceed the amplifier saturation voltage, and then goes by the adaptive pre-distortion amplifier system. The method combined pre-distortion compared with only pre-distortion can be good to compensation of the amplitude distortion so that constellation points do not deflect. It is not obvious that the diffusion of the constellation points of combined model have a less extent.

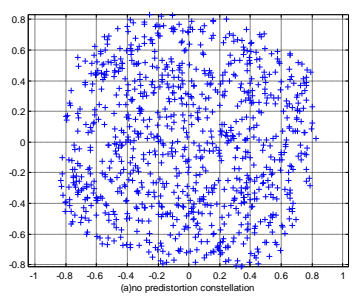

(a)

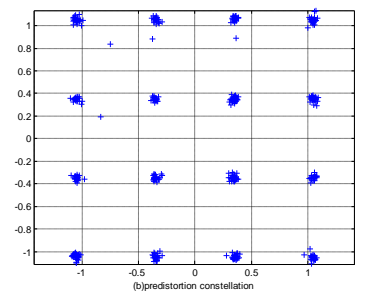

(b)
Figure 4. Constellation:(a) no pre-distortion, (b) pre-distortion

\section{B. Power spectral density}

The power spectral density in combined model is shown in Fig 5, and compared with the clipping and signal of power spectrum in the traditional pre-distortion systems. From the figure, we can see the spectrum of signal about the improved clipping method and the original spectrum are substantially the same, indicating that the algorithm can effectively suppress the spectral band. After pre-distortion combined PAPR suppression treatment, compared with signal directly into the amplifier, band spread spectrum improve about $10 \mathrm{~dB}$, described technical performance of PAPR suppression combined pre-distortion is slightly better than the traditional pre- distortion.

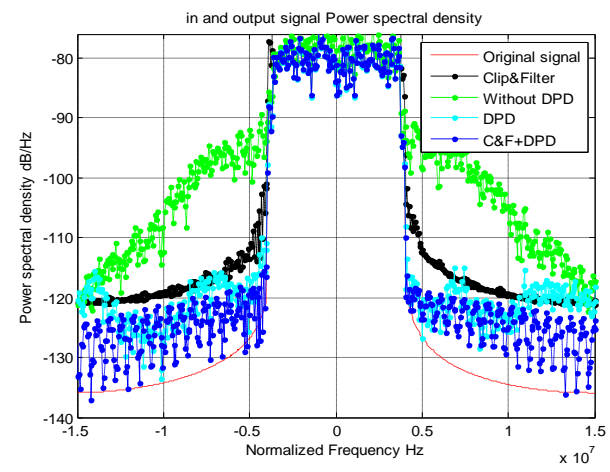

Figure 5. Signal power spectral density 


\section{Clipping and filtering}

Fig 6 shows the comparison of the use of clipping and filtering techniques of BER performance. Where the "C" represents the only case of clipping, the "C\&F" represents the case of clipping and filtering. Seen from the figure, when the value of CR (clipping ratio) decreases, the BER performance deteriorated, showing that the improved method of clipping and filtering method than the clipping performance improves.

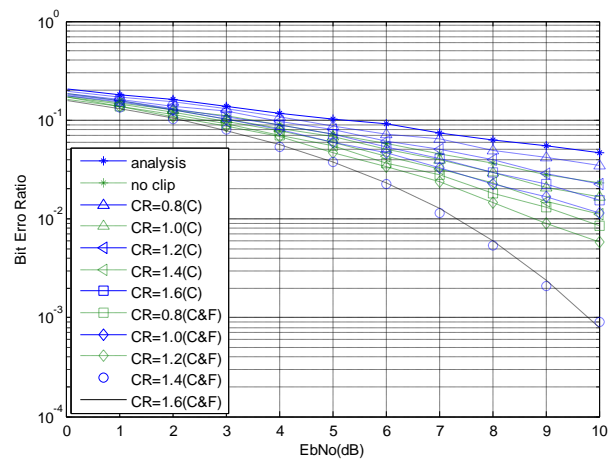

Figure 6. BER performance of different clipping ratio

\section{BER performance}

Fig 7 shows the comparison of the BER performance of using pre-distortion and clipping combined pre-distortion method. Then, we can make deep analysis about system performance under the joint approach form the BER. The figure shows the bit error rate performance of the system when the SNR ranges from 0 to 18 . When the value of SNR equals 10 and the value of $\mathrm{CR}$ equals 0.8 , the value of binary bit error rate is about $10^{-1}$. However, when the value of CR equals and without any processing, the value of predistortion BER is close to $10^{-3}$. In the combined mode system, the value of BER is also close to $10^{-5}$, improving the system's performance.

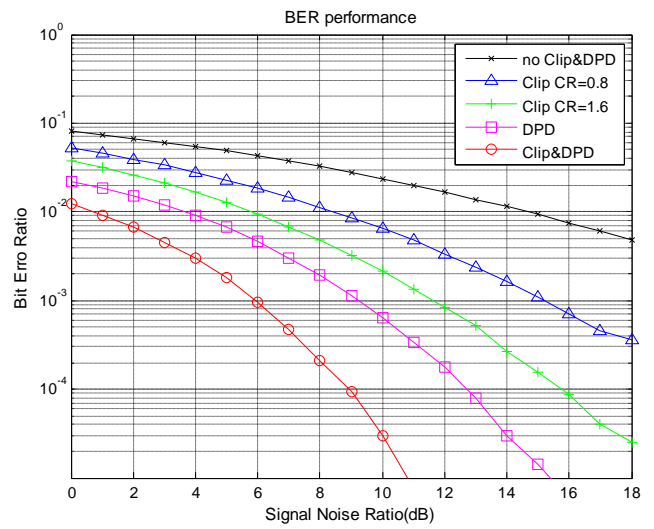

Figure 7. BER performance clipping and pre-distortion

\section{CONCLUSION}

Seen from the simulation result in this paper, if there is a suitable clipping about the amplitude threshold, the impact on system performance can be controlled in a small range, lowering into the PAPR of the amplifier pre-distortion signal. Simulation results show that PAPR suppression combined pre-distortion technique can be well suppressed with the distortion and band spread spectrum, and its performance is better than only using pre-distortion performance alone.

\section{REFERENCES}

[1] Fazel K, Kaiser S. Multi-carrier and spread spectrum systems: from OFDM and MC-CDMA to LTE and WiMAX[M]. Wiley. com, 2008.

[2] Han S H, Lee J H. An overview of peak-to-average power ratio reduction techniques for multicarrier transmission[J]. Wireless Communications, IEEE, 2005, 12(2): 56-65.

[3] Garg R. An adaptive lineariser for high power amplifiers[J]. 2012.

[4] Niranjan M, Srikanth S. Adaptive active constellation extension for PAPR reduction in OFDM systems[C]//Recent Trends in Information Technology (ICRTIT), 2011 International Conference on. IEEE, 2011: 1186-1189.

[5] Bai E W. Frequency domain identification of Hammerstein models $[\mathrm{M}] / /$ Block-oriented Nonlinear System Identification. Springer London, 2010: 161-180.

[6] Bo A, Zhi-xing Y, Chang-yong P, et al. Effects of PAPR reduction on HPA predistortion[J]. Consumer Electronics, IEEE Transactions on, 2005, 51(4): 1143-1147.

[7] Zayani R, Bouallegue R. Pre-Distortion for the compensation of HPA nonlinearity with neural networks: Application to satellite communications[J]. Int. J. Comput. Sci. Netw. Secur, 2007, 7(3): 97 103.

[8] Li H, Kwon D H, Chen D, et al. A fast digital predistortion algorithm for radio-frequency power amplifier linearization with loop delay compensation[J]. Selected Topics in Signal Processing, IEEE Journal of, 2009, 3(3): 374-383.

[9] Chow C W, Yeh C H, Wang C H, et al. Signal remodulation of OFDM-QAM for long reach carrier distributed passive optical networks[J]. Photonics Technology Letters, IEEE, 2009, 21(11): 715717.

[10] Ryu H G, Hoa T P, Lee K M, et al. Improvement of power efficiency of HPA by the PAPR reduction and predistortion[J]. Consumer Electronics, IEEE Transactions on, 2004, 50(1): 119-124. 